\title{
The Relationship Between Initial Trunk Performances and Functional Prognosis in Patients With Stroke
}

\author{
Tha Joo Kim, MD, Kyung Mook Seo, MD, PhD, Don-Kyu Kim, MD, PhD, Si Hyun Kang, MD, PhD \\ Department of Physical Medicine and Rehabilitation, Chung-Ang University College of Medicine, Seoul, Korea
}

\begin{abstract}
Objective To confirm the relationship between initial trunk performance and functional outcomes according to gait ability, and whether initial trunk performance is of predictive value in terms of functional prognosis in patients with stroke.

Methods We reviewed 135 patients who suffered from stroke. Trunk performance of the patients was evaluated using the Trunk Impairment Scale (TIS). The patients were divided into 2 groups according to gait ability at initial stage of stroke. Correlation analyses were performed to assess relationship between initial TIS and functional outcomes. We also evaluated the relationship between initial TIS and the Korean version of Modified Barthel Index (K-MBI) subitems. Finally, stepwise multiple regression analyses were performed to examine the predictive validity of initial TIS and its subscales with functional outcomes.

Results For both groups, initial TIS was correlated with K-MBI and Functional Ambulation Categories at 4 weeks after stroke; however, the relationship did not remain stable at 6 months in ambulatory patients. All K-MBI subitems, which were associated with trunk movement, as well as others about basic skills was correlated with initial TIS. Finally, when of subscales TIS, dynamic sitting balance (TIS-D) was included in by stepwise multiple regression analyses, high proportion of the explained variance was represented.

Conclusion The strong relationship between trunk performance and functional outcomes in patients with stroke emphasizes the importance of trunk rehabilitation. Indeed, an evaluation of a patient's initial TIS after stroke, especially TIS-D, could be helpful in predicting patient's functional prognosis.
\end{abstract}

Keywords Stroke, Postural balance, Gait, Prognosis, Activities of daily living

Received June 18, 2014; Accepted September 4, 2014

Corresponding author: Kyung Mook Seo

Department of Physical Medicine and Rehabilitation, Chung-Ang University College of Medicine, 102 Heukseok-ro, Dongjak-gu, Seoul 156755 , Korea

Tel: +82-2-6299-1882, Fax: +82-2-6298-1866, E-mail: kmseo@cau.ac.kr

(c) This is an open-access article distributed under the terms of the Creative Commons Attribution Non-Commercial License (http://creativecommons. org/licenses/by-nc/3.0) which permits unrestricted noncommercial use, distribution, and reproduction in any medium, provided the original work is properly cited.

Copyright () 2015 by Korean Academy of Rehabilitation Medicine

\section{INTRODUCTION}

Gait disturbance(s) after suffering a stroke may significantly affect one's daily activities. Thus, assessment of a patient's gait ability immediately after a stroke and establishment of a treatment plan are most important before beginning rehabilitation. Gait ability is closely associated not only with muscle strength of upper and lower extremities of the clinically affected side, but also with 
balance control. Balance impairment may cause not only an extension of one's hospitalization after stroke, but also a deterioration of the motility and functional level [1]. Many studies have reported that balance control was a significant clinical marker for predicting overall prognosis of patients at initial stages of stroke [2-4]. Indeed, although standing posture is impossible in most patients at the initial stage of stroke, most previous studies on balance of hemiplegic patients after stroke mainly focused on standing balance. The Berg Balance Scale, which is currently widely used to assess balance and gait ability of patients with stroke, consists of items closely related to daily activities; it has also been proven to be closely associated with prognosis of patients with stroke. However, despite its usefulness, limitations, such as low sensitivity, inadequate items for assessing sitting balance, and the ceiling effect, have also been reported [5-7].

Many researchers suggested development of an assessment method to objectify sitting balance via clinical assessment. However, many methods showed a methodological limitation by simply reflecting balance maintenance time rather than functional assessment [8]. Verheyden et al. [9] presented the concept of trunk performance, which is closely associated with actual activities, including sitting balance; they developed the Trunk Impairment Scale (TIS) to objectively assess trunk performance objectively. They validated reliability and internal consistency of TIS and confirmed the correlation of TIS with sitting balance, gait, and functional ability in patients with stroke $[10,11]$. The TIS provides a convenient assessment and is more advantageous than previous assessment tools as it can provide a more classified assessment due to a less pronounced ceiling effect [9]. Reports have indicated that the assessment of patient's trunk performance via TIS reflected the functional level of patients with stroke in the chronic stage better than other typically-used variables, such as early Korean version of Modified Barthel Index (K-MBI), the patient's age, and the time required for hospitalization, which are known to be correlated with the functional prognosis of patients with stroke $[9,12,13]$. However, these studies had numerous limitations. The study was conducted on some patients with stroke in the chronic stages, the patients did receive continuous follow-up. Furthermore, although the clinical usefulness of trunk performance may vary depending on the condition of patients with stroke, and in particular, the clinical importance of trunk performance may vary depending on the gait ability at the initial stage of stroke, TIS was assessed without consideration to patients' condition. In addition, only a few studies have been conducted for TIS as a predictor of functional prognosis after stroke.

This study aimed to investigate the relationship between initial trunk performance and functional outcomes according to gait ability, and whether initial trunk performance is of predictive value in terms of functional prognosis in patients with stroke.

\section{MATERIALS AND METHODS}

\section{Subjects}

Of the total patients who were referred to the Department of Rehabilitation after suffering from stroke from February 2009 to February 2013, 135 patients were included in the study; their medical records were reviewed retrospectively. There were two main inclusion criteria for this study: 1) first stroke attack, either cerebral infarct or cerebral hemorrhage and 2) undergoing rehabilitation at our institution and having follow-up evaluation at 6 months post-stroke. Patients were also asked, and confirmed with medical records, whether they had any functional problem before stroke. The following were exclusion criteria for this study: severe cognitive impairment, severe aphasia, severe medical disease, neurological defect other than stroke, severe hemispatial neglect, vestibular organ disorder, orthopedic disease, or musculoskeletal pain at the time of transfer. Patients who had had factors affecting the functional outcome during followup evaluation were also excluded from the study.

\section{Methods}

Clinical assessments were used to evaluate the disability of patients after stroke. The Glasgow Coma Scale was used to evaluate the mental state of the patient; the Korean version of Mini-Mental State Examination (MMSE-K) was use to evaluate cognitive function. The patients' swallowing functions were evaluated through videofluoroscopic swallowing study, and dysphagia was diagnosed when significant abnormal movement was observed on any food or fluid consistency in oral, pharyngeal, and esophageal phases with more than score of 2 on penetration-aspiration scale [14]. The patients' 
voiding functions were confirmed by the patients' voiding diary based on the patients' or care givers' records. Bladder dysfunction was defined when symptoms such as frequency, urgency, incontinence and retention that occurred only after the stroke in previously symptomless patients and the patients' need for indwelling catheter or medications for voiding at the time of the evaluation. Activities of daily living (ADL) was evaluated by K-MBI, confirmed reliability and validity by Jung et al. [15]. And gait function was evaluated by Functional Ambulation Categories (FAC). This 6-point scale assesses ambulation status by determining how much human support the patient requires when walking, regardless of whether or not they use a personal assistive device. FAC does not evaluate endurance, as the patient is only required to walk approximately $10 \mathrm{ft}$ [16]. Trunk performance was evaluated by means of TIS. TIS consists of 3 subscales with a total score of 23, including static sitting balance (TIS-S), dynamic sitting balance (TIS-D), and coordination (TIS-C). TIS-S consists of 3 questions with a total score of 7 . TISD consists of 10 questions with a total score of 10 . TIS-C consists of 4 questions with a total score of 6 . The higher score is, the better trunk balance [9].

To investigate the clinical usefulness of TIS according to gait ability, the patients were divided into two groups: group $1(n=54)$ for patients with partial gait ability and group $2(n=81)$ for patients that had no gait ability at the time of transfer. Group allocation was performed according to FAC score: a dependent ambulator who requires assistance from another person involved in group I (FAC $0-2)$, an independent ambulator who can walk without any support involved in group II (FAC 3-5). K-MBI and FAC of the two groups were compared at two different time periods during rehabilitation- 4 weeks and 6 months after suffering a stroke. In addition, the initial TIS of the two groups and its subscales were compared to assess if there was a statistically significant difference between the two groups. The initial TIS and subscales were analyzed for the relation with K-MBI and FAC obtained 4 weeks and 6 months after stroke. Initial TIS and subscales were analyzed with subitems of K-MBI obtained 4 weeks and 6 months after stroke in the two groups.

Numerous demographic and clinical variables including patient's age, gender, MMSE, GDS, K-MBI, dysphagia, voiding dysfunction that were determined to affect prognosis of patients with stroke in previous studies were also compared with initial TIS in order to investigate the potential use of TIS as a functional prognostic factor of stroke. The subjects of analyses confined to patients of group II, because there was no significant relationship for initial TIS with K-MBI and FAC in group I. The Institutional Review Board at Chung-Ang University Hospital approved the protocol of this study.

\section{Statistical analysis}

Statistical analysis was performed using SPSS ver. 12.0 (SPSS Inc., Chicago, IL, USA). An independent Student t-test was conducted for comparative analysis of the two groups. Pearson correlation analysis was conducted to analyze correlation of TIS and its subscales with K-MBI and FAC. The same method was used to analyze correlation of TIS with K-MBI subitems. Stepwise multiple linear regression analysis was also performed to analyze the predictors that affect K-MBI obtained 4 weeks and 6 months after stroke. Results were considered to be statistically significant at $\mathrm{p}<0.05$.

\section{RESULTS}

\section{General characteristics}

General demographic characteristics of patients are listed in Table 1. Of the total 135 patients, 83 of them were males and 52 females; patients mean age was $62.14 \pm 12.91$ years. The patients included 69 patients who suffered a hemorrhagic stroke, 66 patients with ischemic stroke, 81 patients with right-sided hemiplegia after stroke, and 54 patients with left-sided hemiplegia after stroke. A mean time period of $17.14 \pm 2.51$ days passed until patients were referred after stroke.

There were no significant differences in the two groups for the following variables at baseline: age, gender, type of stroke, affected side, time of transfer, MMSE-K, and GDS. However, the variables K-MBI and FAC were significantly different $(\mathrm{p}<0.05)$ between the two groups at baseline. The value of K-MBI of group I was assessed to be $65.44 \pm 18.59,78.08 \pm 14.41$, and $78.95 \pm 12.49$ at baseline, 4 weeks, and 6 months after stroke rehabilitation, respectively. Meanwhile, K-MBI of group II was assessed to be $22.13 \pm 11.17,38.12 \pm 12.92$, and $48.56 \pm 13.96$, respectively. FAC was assessed to be $3.69 \pm 1.12$ and $0.84 \pm 1.58$ in both groups at the time of transfer, $3.91 \pm 0.94$ and $1.42 \pm 1.28$ at 4 weeks after stroke, and $4.04 \pm 1.05$ and $1.91 \pm 1.57$ at 6 months after stroke. These results showed that K-MBI and FAC was significantly higher in group II patients than 
Table 1. Demographic characteristics and clinical variables of study participants

\begin{tabular}{|lccc}
\hline \multicolumn{1}{c}{ Characteristic } & Total $(\mathbf{n}=\mathbf{1 3 5})$ & Group I $(\mathbf{n}=\mathbf{5 4})$ & Group II $(\mathbf{n = 8 1})$ \\
\hline Age (yr) & $62.14 \pm 12.91$ & $60.45 \pm 9.86$ & $63.42 \pm 12.30$ \\
\hline Gender (male:female) & $83: 52$ & $33: 21$ & $50: 31$ \\
\hline Type of stroke (ischemic:hemorrhagic) & $69: 66$ & $22: 32$ & $47: 34$ \\
\hline Affected side (Rt:Lt) & $81: 54$ & $36: 18$ & $45: 36$ \\
\hline Time of referral (day) & $17.14 \pm 2.51$ & $16.23 \pm 4.01$ & $18.40 \pm 2.35$ \\
\hline MMSE & $21.03 \pm 8.66$ & $21.75 \pm 6.28$ & $20.13 \pm 9.30$ \\
\hline GDS & $3.16 \pm 1.34$ & $2.85 \pm 0.84$ & $3.23 \pm 1.31$ \\
\hline Dysphagia*(yes:no) & $50: 85$ & $13: 41$ & $37: 44$ \\
\hline Voiding difficulty* (yes:no) & $27: 108$ & $4: 54$ & $23: 58$ \\
\hline K-MBI & & & \\
\hline Initial* & $38.70 \pm 29.02$ & $65.44 \pm 18.59$ & $22.13 \pm 11.17$ \\
\hline After 4 weeks* & $54.90 \pm 31.67$ & $78.08 \pm 14.41$ & $38.12 \pm 12.92$ \\
\hline After 6 months* & $60.86 \pm 27.18$ & $78.95 \pm 12.49$ & $48.56 \pm 13.96$ \\
\hline FAC & & & \\
\hline Initial & & $3.69 \pm 1.12$ & $0.84 \pm 1.58$ \\
\hline After 4 weeks* & $1.29 \pm 1.34$ & $3.91 \pm 0.94$ & $1.42 \pm 1.28$ \\
\hline After 6 months* & $2.12 \pm 1.49$ & $4.04 \pm 1.05$ & $1.91 \pm 1.57$ \\
\hline TIS & $3.15 \pm 1.60$ & & $8.19 \pm 4.34$ \\
\hline Total* & & $14.37 \pm 4.61$ & $3.68 \pm 2.58$ \\
\hline TIS-S* & $9.53 \pm 5.28$ & $6.31 \pm 2.45$ & $4.21 \pm 2.69$ \\
\hline TIS-D* & $5.29 \pm 4.02$ & $7.23 \pm 2.68$ & $1.21 \pm 1.13$ \\
\hline TIS-C* & $4.14 \pm 3.18$ & $2.75 \pm 1.77$ & \\
\hline
\end{tabular}

Values are presented as mean \pm standard deviation or numbers of patients.

Group I, ambulatory patients after stroke; Group II, non-ambulatory patients after stroke; MMSE, Mini-Mental State Examination; GDS, Global Deterioration Scale; K-MBI, Korean version of Modified Barthel Index; FAC, Functional Ambulation Categories; TIS, Trunk Impairment Scale; TIS-S, static sitting balance subscale of TIS; TIS-D, dynamic sitting balance subscale of TIS; TIS-C, coordination subscale of TIS.

${ }^{*} \mathrm{p}<0.05$.

group II patients for all the time points measured.

Initial TIS at the time of transfer was assessed to be $14.37 \pm 4.61$ in the group I and $8.19 \pm 4.34$ in the group II, showing a significantly higher score in group I. TIS-S was assessed to be $6.31 \pm 2.45$ and $3.68 \pm 2.58$ in each group, respectively. TIS-D was assessed to be $7.23 \pm 2.68$ and $4.21 \pm 2.69$ and TIS-C was assessed to be $2.75 \pm 1.77$ and $1.21 \pm 1.13$ in each group, respectively. The results showed that initial TIS and its subscales were all significantly higher in ambulatory patients than in non-ambulatory patients $(\mathrm{p}<0.05)($ Table 1$)$.

\section{Correlation of initial TIS with K-MBI and FAC}

The results of correlation analysis between initial TIS and functional outcomes are summarized in Table 2. In group I, K-MBI obtained at 4 weeks after stroke was assessed to have a correlation coefficient $(\mathrm{r}=0.418, \mathrm{p}<0.05)$ for initial TIS, a correlation coefficient $(r=0.415, p<0.05)$ for TIS-D, and a correlation coefficient $(\mathrm{r}=0.334, \mathrm{p}<0.05)$ for TIS-C; all of these correlations were statistically significant. However, there was no significant correlation was shown for TIS-S. K-MBI obtained at 6 months after stroke showed no significant correlation with initial TIS and its subscales. Furthermore, FAC obtained at 4 weeks and 6 months after stroke showed no significant correlation with initial TIS and its subscales.

In group II, K-MBI obtained at 4 weeks and 6 months after stroke was assessed to be correlated with initial TISK-MBI obtained at 4 weeks after stroke was assessed to have a correlation coefficient $(r=0.558, p<0.05)$ for initial 
Table 2. Correlation between initial TIS and functional outcomes

\begin{tabular}{|c|c|c|c|c|c|c|c|c|}
\hline & \multicolumn{4}{|c|}{ Group I } & \multicolumn{4}{|c|}{ Group II } \\
\hline & TIS & TIS-S & TIS-D & TIS-C & TIS & TIS-S & TIS-D & TIS-C \\
\hline \multicolumn{9}{|l|}{ K-MBI } \\
\hline After 4 weeks & $0.418^{*}$ & 0.159 & $0.415^{*}$ & $0.334^{*}$ & $0.558^{*}$ & $0.376^{*}$ & $0.562^{*}$ & 0.231 \\
\hline After 6 months & 0.008 & -0.105 & -0.069 & 0.259 & $0.439 *$ & $0.438^{*}$ & $0.464^{*}$ & 0.252 \\
\hline \multicolumn{9}{|l|}{ FAC } \\
\hline After 4 weeks & 0.253 & -0.016 & 0.269 & 0.130 & $0.491^{*}$ & $0.320^{*}$ & $0.492^{*}$ & 0.131 \\
\hline After 6 months & 0.277 & 0.092 & 0.165 & 0.554 & $0.453^{*}$ & $0.434^{*}$ & $0.441^{*}$ & 0.218 \\
\hline
\end{tabular}

Group I, ambulatory patients after stroke; Group II, non-ambulatory patients after stroke; TIS, Trunk Impairment Scale; TIS-S, static sitting balance subscale of TIS; TIS-D, dynamic sitting balance subscale of TIS; TIS-C, coordination subscale of TIS; K-MBI, Korean version of Modified Barthel Index; FAC, Functional Ambulation Categories. ${ }^{*} \mathrm{p}<0.05$.

Table 3. Correlation between initial TIS and subitems of K-MBI in non-ambulatory patients with stroke

\begin{tabular}{|lllll|}
\hline & TIS & TIS-S & TIS-D & TIS-C \\
\hline K-MBI after 4 weeks & & & & \\
\hline Feeding & $0.451^{*}$ & $0.126^{*}$ & $0.462^{*}$ & 0.206 \\
\hline Bathing & $0.352^{*}$ & $0.345^{*}$ & $0.215^{*}$ & $0.295^{*}$ \\
\hline Grooming & $0.261^{*}$ & $0.192^{*}$ & $0.215^{*}$ & 0.111 \\
\hline Dressing & $0.265^{*}$ & $0.265^{*}$ & $0.163^{*}$ & 0.091 \\
\hline Bowel control & $0.385^{*}$ & $0.335^{*}$ & $0.358^{*}$ & 0.333 \\
\hline Bladder control & $0.421^{*}$ & $0.368^{*}$ & $0.492^{*}$ & 0.342 \\
\hline Toilet use & $0.559^{*}$ & $0.356^{*}$ & $0.567^{*}$ & $0.372^{*}$ \\
\hline Chair/bed transfer & $0.576^{*}$ & $0.261^{*}$ & $0.551^{*}$ & $0.360^{*}$ \\
\hline Mobility/ambulation & $0.506^{*}$ & $0.256^{*}$ & $0.399^{*}$ & $0.301^{*}$ \\
\hline Stair climbing & $0.421^{*}$ & 0.179 & $0.391^{*}$ & 0.306 \\
\hline K-MBI after 6 months & & & & 0.265 \\
\hline Feeding & $0.365^{*}$ & $0.265^{*}$ & $0.296^{*}$ & $0.245^{*}$ \\
\hline Bathing & $0.226^{*}$ & $0.496^{*}$ & $0.265^{*}$ & 0.365 \\
\hline Grooming & $0.244^{*}$ & $0.325^{*}$ & $0.245^{*}$ & 0.035 \\
\hline Dressing & $0.264^{*}$ & $0.156^{*}$ & $0.165^{*}$ & 0.423 \\
\hline Bowel control & $0.573^{*}$ & $0.510^{*}$ & $0.610^{*}$ & 0.131 \\
\hline Bladder control & $0.442^{*}$ & $0.448^{*}$ & $0.436^{*}$ & $0.242^{*}$ \\
\hline Toilet use & $0.446^{*}$ & $0.403^{*}$ & $0.449^{*}$ & $0.222^{*}$ \\
\hline Chair/bed transfer & $0.461^{*}$ & $0.499^{*}$ & $0.472^{*}$ & $0.281^{*}$ \\
\hline Mobility/ambulation & $0.382^{*}$ & $0.310^{*}$ & $0.373^{*}$ & 0.312 \\
\hline Stair climbing & $0.377^{*}$ & $0.351^{*}$ & $0.406^{*}$ & 5458 \\
\hline
\end{tabular}

TIS, Trunk Impairment Scale; TIS-S, static sitting balance subscale of TIS; TIS-D, dynamic sitting balance subscale of TIS; TIS-C, coordination subscale of TIS; K-MBI, Korean version of Modified Barthel Index; FAC, Functional Ambulation Categories.

${ }^{*} \mathrm{p}<0.05$.

TIS, $r=0.376(p<0.05)$ for TIS-S, and $r=0.562(p<0.05)$ for TIS-D; K-MBI obtained at 6 months after stroke was assessed to have $r=0.439(p<0.05)$ for initial TIS, $r=0.438$ $(\mathrm{p}<0.05)$ for TIS-S, and $\mathrm{r}=0.464(\mathrm{p}<0.05)$ for TIS-D. FAC obtained at 4 weeks after stroke was assessed to have $\mathrm{r}=0.491(\mathrm{p}<0.05)$ for initial TIS, $\mathrm{r}=0.320(\mathrm{p}<0.05)$ for TIS-S, 
Table 4. Stepwise multiple linear regression analysis of initial TIS for K-MBI in non-ambulatory patients with stroke

\begin{tabular}{lccccc}
\hline & df & $\boldsymbol{\beta}$ & p-value & $\mathbf{R}^{\mathbf{2}}$ & $\mathbf{F}$ \\
\hline K-MBI after 4 weeks & & & & & \\
(Constant) & 8.446 & - & - & 0.669 & 59.997 \\
K-MBI (initial) & - & 0.441 & $0.019^{*}$ & - & - \\
MMSE & - & 0.165 & $0.049^{*}$ & - & - \\
TIS-D & - & 0.534 & $<0.001^{*}$ & - & - \\
K-MBI after 6 months & & & & & \\
(Constant) & 8.148 & - & - & 0.653 & 213.767 \\
TIS-D & - & 0.568 & $<0.001^{*}$ & - & - \\
\hline
\end{tabular}

K-MBI, Korean version of Modified Barthel Index; MMSE, Mini-Mental State Examination; TIS, Trunk Impairment Scale; TIS-D, dynamic sitting balance subscale of TIS.

${ }^{*} \mathrm{p}<0.05$.

and $\mathrm{r}=0.492(\mathrm{p}<0.05)$ for TIS-D; FAC obtained at 6 months after stroke was assessed to have $\mathrm{r}=0.453(\mathrm{p}<0.05)$ for initial TIS, $\mathrm{r}=0.434(\mathrm{p}<0.05)$ for TIS-S, and $\mathrm{r}=0.441(\mathrm{p}<0.05)$ for TIS-D. TIS-C was showed no significant correlation with K-MBI and FAC for all the time points measured.

\section{Correlation of TIS with subitems of K-MBI after stroke} non-ambulatory patients

The results of the correlation analysis between initial TIS and subitems of K-MBI in non-ambulatory patients are summarized in Table 3. In group II, initial TIS and most of TIS subscales were positively correlated with 10 subitems of K-MBI at 4 weeks after stroke; there was a significant correlation $(\mathrm{p}<0.05)$. However, TIS-C showed a significant correlation with only 5 subitems of K-MBI at 4 weeks after stroke: bathing, toilet use, chair/bed transfer, and mobility/ambulation. This relationship between TIS-C and K-MBI subitems in general remained stable at 6 months after suffering a stroke (Table 3).

Stepwise multiple linear regression analysis of initial TIS for K-MBI in non-ambulatory patients

The results of the stepwise multiple regression analysis for K-MBI obtained at 4 weeks and 6 months after suffering a stroke are summarized in Table 4 . The model with the highest explained variance for K-MBI at 4 weeks after stroke was presented when TIS-D was included $\left(\mathrm{R}^{2}=0.669\right)$. The most significantly correlated factors of the model were shown to be TIS- $D(\beta=0.534, p<0.001)$, initial K-MBI $(\beta=0.441, p=0.019)$ and MMSE $(\beta=0.165$, $\mathrm{p}<0.049)$ for K-MBI obtained at 4 weeks after stroke.

TIS-D was shown to be the only significant factor for K-
MBI obtained at 6 months after stroke $\left(R^{2}=0.653, \beta=0.568\right.$, $\mathrm{p}<0.001)$. Patients' age, gender, GDS, dysphagia, and voiding difficulty were not included as significant factors on regression equation for K-MBI obtained at 6 months.

\section{DISCUSSION}

This study aimed to investigate the clinical importance of TIS according to gait ability in patients with stroke, as well as to confirm the relationship of initial TIS and functional prognosis of rehabilitation. Prognosis prediction via clinical assessment has been investigated by myriad researchers, thanks to easy conduct without a particular device [17-19]. Many studies have reported that a patient's sitting balance in initial stage after stroke was closely related to the functional level of patients. These studies emphasized that sitting balance should be the focus of clinical examination and statistical analysis. Recently, new methods have been offered to measure and assess sitting balance: the trunk control test (TCT), Postural Assessment Scale for Stroke-trunk control (PASSTC), and TIS have been the most commonly used instruments as tools to assess trunk balance performance. TCT assesses a patient's performance in both lying and sitting posture; both have been validated to be related to the recovery and functional level of the muscle strength of the upper and lower extremities. However, TCT poorly reflects the quality of movement, and is weakly related to the actual movement of the trunk muscle. Furthermore, the 'ceiling effect' cannot be ruled out as TCT consists of items easily conducted by patients with stroke $[1,2]$. PASS-TC was developed by selecting five items of trunk 
balance maintenance. The instrument was validated to be used as a tool to assess patient's performance in daily activities. However, PASS-TC poorly reflects the functional level of chronic patients with stroke, and the ceiling effect cannot be ruled out.

Verheyden et al. [9] suggested that the conventional assessment methods poorly reflected trunk performance, which is actually used in daily activities, although trunk performance after stroke significantly affects daily activities, suggesting the TIS as a new assessment method. And it is suggested that each TIS subscale as well as total TIS had sufficient reliability and validity for use in clinical practice.

In this study, initial TIS and functional outcomes at 4 weeks and 6 months after stroke were significantly higher in ambulatory patients than in non-ambulatory patients. However, initial TIS assessed in ambulatory patients was no significant correlation with K-MBI at 6 months after stroke. It was suggested that trunk performance was not integral factor for functional outcomes if patients' gait ability was preserved after stroke. In addition, of the TIS subscales, TIS-S showed no significant correlation with K-BMI at 4 weeks after stroke. Although Verheyden et al. [9] suggested TIS as a tool with no ceiling effect, the results of this study showed that ceiling effect was not ruled out in the assessment of TIS-S in ambulatory patients, with 34 patients (62.97\%) scoring 7 (maximum score) and 16 patients $(29.63 \%)$ scoring 6 . This is likely to be attributable to the fact that total score of initial TIS was not correlated with K-MBI at 6 month after stroke. However, the additional studies is needed to support this result and clinical implication of trunk performance in ambulatory patients after stroke should be confirmed by means of various objective measurements, such as isokinetic muscle testing, EMG analysis or motion analysis

To investigate the relationship of TIS with performance in daily activities, K-MBI subitems were analyzed for their correlation with initial TIS. In previous studies, it had been suggested that sitting balance is a crucial component to perform ADL and it might be because K-MBI assess ADL, which requires sitting balance for feeding and dressing. However, as a result of this study, all of KMBI subitems had a significant correlation with initial TIS, such as bowel and bladder control, which is not related with trunk movement, and this relationship was remained over the time course. That is trunk performance have relevance to general performance of ADL as well as a specific motion. Of the TIS subscales, TIS-C showed a significant correlation with only 5 K-MBI subitems for all the time points measured and these subitems which had a significant correlation for K-MBI had little in common feature. It suggested that floor effect could not exclude in TIS-C of non-ambulatory patients, with 36 patients (44.45\%) scoring 0 or scoring 1 . This should be confirmed in future studies.

Of the TIS subscales, TIS-S evaluates if a patient can maintain an upright sitting position with and without the legs crossed. Because of ADL are performed from a seated position, static sitting balance could be seen as a prerequisite condition. In previous study, it was considered as a significant predictor for functional outcome [12]. However, in our study, even if participants were limited to non-ambulatory patients after stroke, TIS-D was the most important factor when predicting K-MBI and FAC at 4 weeks and 6 months after stroke. TIS-D examines the ability to perform lateral flexion of trunk and reflected dynamic trunk movement. Eventually it might be more essential for performing ADL than static trunk control and supports the importance of active trunk rehabilitation.

This study had the following limitations. First, our study was conducted retrospectively, using the medical records of subject patients. For this reason, bias in patient selection cannot be ruled out. Second, limited assessment tools were used to assess the patients' functional independence and gait level. Although K-MBI and FAC have been widely used in patients with stroke, and their reliability and eligibility have already been validated [8], other assessment tools, such as the trunk control test, are required for comparison to show the usefulness of TIS. Nonetheless, the results of our study again confirmed the clinical usefulness of TIS, particularly according to gait ability after stroke. In addition, this study is meaningful as it was conducted to investigate which is considered as predictor of ADL and gait function for the establishment of a better rehabilitation program.

In conclusion, the strong relationship between trunk performance and functional outcomes in patients with stroke emphasizes the importance of trunk rehabilitation. In particular, clinicians should evaluate initial TIS after stroke, especially TIS-D; this can be used as a useful predictor of performance in functional prognosis in patients 
with stroke. However, the application of TIS in clinical practice and stroke research is needed consideration on patient's gait ability and severity of trunk impairment.

\section{CONFLICT OF INTEREST}

No potential conflict of interest relevant to this article was reported.

\section{REFERENCES}

1. Michael KM, Allen JK, Macko RF. Reduced ambulatory activity after stroke: the role of balance, gait, and cardiovascular fitness. Arch Phys Med Rehabil 2005;86:1552-6.

2. Verheyden G, Nieuwboer A, De Wit L, Thijs V, Dobbelaere J, Devos H, et al. Time course of trunk, arm, leg, and functional recovery after ischemic stroke. Neurorehabil Neural Repair 2008;22:173-9.

3. Sandin KJ, Smith BS. The measure of balance in sitting in stroke rehabilitation prognosis. Stroke 1990;21:82-6.

4. Feigin L, Sharon B, Czaczkes B, Rosin AJ. Sitting equilibrium 2 weeks after a stroke can predict the walking ability after 6 months. Gerontology 1996;42:348-53.

5. Amusat N. Assessment of sitting balance of patients with stroke undergoing inpatient rehabilitation. Physiother Theory Pract 2009;25:138-44.

6. Berg K. Measuring balance in the elderly: preliminary development of an instrument. Physiother Can 1989;41:304-11.

7. Blum L, Korner-Bitensky N. Usefulness of the Berg Balance Scale in stroke rehabilitation: a systematic review. Phys Ther 2008;88:559-66.

8. Nieuwboer A, Feys H, De Weerdt W, Nuyens G, De Corte E. Developing a clinical tool to measure sitting balance after stroke: a reliability study. Physiotherapy 1995;81:439-45.

9. Verheyden G, Nieuwboer A, Mertin J, Preger R, Kiekens $\mathrm{C}$, De Weerdt W. The Trunk Impairment Scale: a new tool to measure motor impairment of the trunk after stroke. Clin Rehabil 2004;18:326-34.

10. Gjelsvik B, Breivik K, Verheyden G, Smedal T, Hofstad $\mathrm{H}$, Strand LI. The Trunk Impairment Scale - modified to ordinal scales in the Norwegian version. Disabil Rehabil 2012;34:1385-95.

11. Heyrman L, Desloovere K, Molenaers G, Verheyden G, Klingels K, Monbaliu E, et al. Clinical characteristics of impaired trunk control in children with spastic cerebral palsy. Res Dev Disabil 2013;34:327-34.

12. Verheyden G, Nieuwboer A, De Wit L, Feys H, Schuback B, Baert I, et al. Trunk performance after stroke: an eye catching predictor of functional outcome. J Neurol Neurosurg Psychiatry 2007;78:694-8.

13. Verheyden G, Vereeck L, Truijen S, Troch M, Herregodts I, Lafosse C, et al. Trunk performance after stroke and the relationship with balance, gait and functional ability. Clin Rehabil 2006;20:451-8.

14. Rosenbek JC, Robbins JA, Roecker EB, Coyle JL, Wood JL. A penetration-aspiration scale. Dysphagia 1996;11:93-8.

15. Jung HY, Park BK, Shin HS, Kang YK, Pyun SB, Paik NJ, et al. Development of the Korean version of Modified Barthel Index (K-MBI): multi-center study for subjects with stroke. J Korean Acad Rehabil Med 2007;31:28397.

16. Holden MK, Gill KM, Magliozzi MR, Nathan J, PiehlBaker L. Clinical gait assessment in the neurologically impaired: reliability and meaningfulness. Phys Ther 1984;64:35-40.

17. Kwakkel G, Wagenaar RC, Kollen BJ, Lankhorst GJ. Predicting disability in stroke: a critical review of the literature. Age Ageing 1996;25:479-89.

18. Loewen SC, Anderson BA. Predictors of stroke outcome using objective measurement scales. Stroke 1990;21:78-81.

19. Ween JE, Alexander MP, D'Esposito M, Roberts M. Factors predictive of stroke outcome in a rehabilitation setting. Neurology 1996;47:388-92. 